\title{
Effect of low-dose lidocaine on objective upper extremity strength and immediate pain relief following cervical interlaminar epidural injections: a double-blinded randomized controlled trial
}

\author{
Zachary L. McCormick, Taylor Burnham, ${ }^{2}$ Shellie Cunningham, ${ }^{2}$ Richard W Kendall, \\ David Bougie, ${ }^{3}$ Masaru Teramoto, ${ }^{2}$ David R. Walega ${ }^{4}$
}

${ }^{1}$ Division of Physical Medicine and Rehabilitation, Department of Orthopedic Surgery, University of Utah Hospital, Salt Lake City, Utah, USA 2Physical Medicine and Rehabilitation, University of Utah Hospital, Salt Lake City, Utah, USA

${ }^{3}$ Department of Anesthesiology, Northwestern University,

Chicago, Illinois, USA

${ }^{4}$ Anesthesiology, Northwestern University Feinberg School of Medicine, Chicago, Illinois, USA

Correspondence to Dr Zachary L. McCormick, Division of Physical Medicine and Rehabilitation, Department of Orthopedic Surgery, University of Utah Hospital, Salt Lake City, UT 84103, USA; zmccormi@gmail.com

Received 27 April 2020 Revised 27 June 2020 Accepted 2 July 2020 Published Online First 11 August 2020

Check for updates

(C) American Society of Regional Anesthesia \& Pain Medicine 2020. No commercial re-use See rights and permissions. Published by BMJ.

To cite: McCormick ZL, Burnham T, Cunningham S, et al. Reg Anesth Pain Med 2020:45:767-773.

\section{ABSTRACT}

Background Low-dose lidocaine is a common diluent for analgesia following cervical interlaminar epidural steroid injection (CIESI). Concerns with this practice exist. A single-arm cohort reported that $20 \%$ of patients develop postprocedural upper extremity weakness when using lidocaine as a diluent. Furthermore, a high-cervical spinal block with unintended intrathecal or subdural administration is possible.

Objective Determine if low-dose lidocaine as a diluent during CIESI causes clinically meaningful (1) upper extremity weakness and (2) immediate pain relief when compared with saline.

Design Double-blinded randomized control trial. Methods Patients with cervical radicular pain scheduled for CIESI were enrolled. Participants received lidocaine (CIESI-L) or saline (CIESI-S) as a diluent for the epidural injectate. Myotomal strength was measured with dynamometry before and between 20 and $30 \mathrm{~min}$ after CIESI. Pre-pain and post-pain scores were obtained. Primary and secondary outcomes were post-CIESI weakness of $\geq 20 \%$ (minimal clinically important difference (MCID)) in $\geq 1$ myotome and $\geq 50 \%$ pain reduction on the numerical scale.

Results 120 participants (64 females (53\%), mean age, 56 (SD 13.7) years) completed the study and were analyzed (CIESI-L $n=60$; CIESI-S $n=60$ ). There was no significant between-group difference in the proportion of participants with postprocedural weakness, CIESI-L $41.7 \%(95 \% \mathrm{Cl}, 29.8 \%$ to $54.5 \%)$, CIESL-S 50\% (95\% $\mathrm{Cl}, 37.5 \%$ to $62.5 \%$ ). Between-group comparison showed no significant difference in pain reduction, relative risk $1.53(95 \% \mathrm{Cl}, 0.82$ to 2.86$)$.

Conclusion Low-dose lidocaine as a diluent in CIESI does not significantly increase the risk of post-CIESI myotomal weakness when compared with saline, but also does not substantially increase the likelihood of immediate, meaningful pain relief.

Trial registration details ClinicalTrials.gov (NCT03127137); December 26, 2017.

\section{INTRODUCTION}

Cervical radicular pain has an incidence of 83 per 100,000 in the US population. ${ }^{1}$ Recalcitrant cervical radicular pain is commonly treated with epidural steroid injections. ${ }^{2}$ Cervical interlaminar epidural steroid injection (CIESI) is the most common technique to access the epidural space..$^{3-6}$ The majority of the literature on the effectiveness of CIESI includes the use of low-dose local anesthetic as a diluent for the steroid injectate with the intention of providing immediate pain relief. ${ }^{7-14}$ While this practice is common, ${ }^{7}$ it is not supported by evidence. Currently, clinical practice guidelines neither recommend nor discourage the use of local anesthetic as a diluent. ${ }^{5}$

There are risks associated with local anesthetic use as a diluent. Local anesthetic unintentionally placed in the intrathecal or subdural space can result in apnea, hypotension, bradycardia, paralysis, and loss of airway reflexes, also known as highspinal block (HSB). ${ }^{8-13}$ Furthermore, previous work demonstrates transient upper extremity weakness after appropriately placed transforaminal delivery of steroids with low-dose local anesthetic. ${ }^{14} \mathrm{We}$ reported similar findings in a single-arm cohort study, wherein $20 \%$ of participants experienced a clinically meaningful decrease in upper extremity strength, as assessed by dynamometry, in at least one myotome after a single CIESI injection with $1.0 \mathrm{~mL}$ of $1 \%$ lidocaine used as a diluent. ${ }^{15}$

Given a lack of clear evidence or a consensus recommendation for the use of local anesthetic as a diluent during CIESI, the present study was designed to determine if low-dose lidocaine used during CIESI results in (1) clinically significant upper extremity weakness and (2) immediate pain relief when compared with saline. We hypothesized that CIESI with lidocaine would cause an objective transient decrease in upper extremity strength and a significant immediate decrease in pain compared with saline. Broadly, we also sought to place our findings into a risk-benefit analysis that includes the risk of $\mathrm{HSB}$, in order to provide evidence to aid clinical decision-making regarding diluent choice for CIESI.

\section{METHODS}

\section{Study design and patient selection}

This double-blinded randomized control trial was conducted at a single urban tertiary academic pain medicine center. Patients were recruited and treated between August 2018 and May 2019. All participants provided written informed consent prior to undergoing any study related procedures. 
Eligible patients were 18 years and older who were scheduled to undergo CIESI for the treatment of cervical radicular pain. Patients were included if they had a clinical diagnosis of cervical radicular pain, a numerical rating scale (NRS) for pain $\geq 4$ at the time of screening, duration of radicular pain $\geq 4$ weeks, and failure of conservative therapy (oral analgesics, oral antiinflammatories, physical therapy). Exclusion criteria included pregnancy, breastfeeding, refusal to participate, allergy or hypersensitivity to steroid or amide local anesthetics, contraindication to CIESI, inability to perform upper extremity strength testing, prior stroke or other neurological or neuromuscular disease, or need for or request of conscious sedation during the CIESI procedure.

\section{Randomization and masking}

A computer-generated 1:1 block randomization scheme (https:// www.randomizer.org) was used to assign participants to receive a CIESI of $80 \mathrm{mg}$ triamcinolone acetonide $(40 \mathrm{mg} / \mathrm{mL})$ with either $2.0 \mathrm{~mL} 1 \%$ lidocaine or $2.0 \mathrm{~mL}$ preservative-free saline. Randomization was performed by the injectionist immediately before the injection procedure by opening an opaque envelope to reveal the participant number and group assignment printed inside of the envelope. Participants and all other study personnel were blinded to group assignment. The interventionalist performing the CIESI was not blinded, but was not involved in any data collection.

\section{Data collection}

Preprocedural baseline demographic, clinical, and imaging information were collected. Age, gender, body mass index (BMI), duration of symptoms, laterality of symptoms, and preprocedural NRS pain scores were recorded by a blinded authorized study team member.

\section{Strength measurements}

Bilateral upper extremity strength testing was performed by a blinded trained research assistant. Bilateral elbow flexion (EF;C5), wrist extension (WE;C6), elbow extension (EX;C7), and handgrip (HG;C8,T1) strength were measured with a push-pull hydraulic dynamometer (Baseline Evaluation Instruments, Somerset, UK) and handgrip strength was evaluated with a digital dynamometer (JAMAR PLUS+, Sammons Preston, Bolingbrook, Illinois, USA). A standard protocol was used to isolate each joint movement and force production. Participants were instructed to offer maximum effort with each exercise. Three baseline strength measurements were recorded for each strength test. Validation studies using similar protocols for upper extremity functional strength measurement show a high degree of test-retest reliability with a narrow range of variability. ${ }^{16-21}$ Measurements were obtained immediately before and between 20 and $30 \mathrm{~min}$ following the procedure.

\section{Procedures}

All CIESI procedures were performed at a single facility by three experienced interventionalists, board-certified in anesthesiology with subspecialty certification in pain medicine.

Participants were placed prone on a fluoroscopy table. A noninvasive blood pressure and ECG monitor, and pulse oximeter were placed. The cervical spine region was cleaned with chlorhexidine and draped in a sterile manner. Fluoroscopy was used to identify the C7-T1 interlaminar space. After injection of 1.0 to $2.0 \mathrm{~mL}$ of $1 \%$ lidocaine to the skin and subcutaneous tissues using a 1.5 -inch $25 \mathrm{G}$ needle, a $17 \mathrm{G} 3.5$-inch Tuohy needle was placed at the C7-T1 level and a loss of resistance (saline) technique was used to gain access to the epidural space. Needle position was confirmed in anterior-posterior and lateral fluoroscopic views. Once a satisfactory target position was achieved, 0.5 to $1.0 \mathrm{~mL}$ of contrast (Omnipaque-180, GE Healthcare, Princeton, New Jersey, USA) was injected under fluoroscopy. On confirmation of a satisfactory epidural contrast pattern, the epidural injectate was delivered. The CIESI-L group received $2.0 \mathrm{~mL}$ of $1 \%$ preservative-free lidocaine and $80 \mathrm{mg}$ triamcinolone acetonide $(40 \mathrm{mg} / \mathrm{mL})$. The CIESI-S group received $2.0 \mathrm{~mL}$ of preservativefree saline and $2.0 \mathrm{~mL}$ of triamcinolone acetonide $(40 \mathrm{mg} / \mathrm{mL})$. In all cases, the total injectate volume was $4.0 \mathrm{~mL}$.

Twenty minutes after the procedure, three measurements for each strength test were recorded. The testing took no more than $10 \mathrm{~min}$ for any individual. The order of muscle testing remained the same for all participants to limit additional variability in the strength measurement protocol. Secondary measures were collected $20 \mathrm{~min}$ after the procedure.

\section{Outcomes}

The primary outcome of this study was the proportion of each group (CIESI-L and CIESI-S) that demonstrated post-CIESI weakness of $\geq 20 \%$ (minimal clinically important difference (MCID)) compared with baseline measures in $\geq 1$ myotome. Strength differences were calculated by subtracting the mean preprocedural and postprocedural CIESI strength measurements for each myotome. The between-group difference was represented by the relative risk (RR), defined as the proportion of participants in the CIESI-L group observed to meet the primary outcome compared with those in the CIESI-S group.

Secondary outcomes included changes in pain score (NRS) and the incidence of adverse events. The proportion of patients in each group (CIESI-L and CIESI-S) that experienced $\geq 50 \%$ immediate pain reduction on the NRS after CIESI was calculated. $\mathrm{A} \geq 50 \%$ pain reduction is a common and recommended categorical outcome measure that reflects a more significant pain reduction than the often-reported MCID. ${ }^{22}$ Adverse events were assessed by questionnaire during postprocedure testing and again during a phone call approximately 1 day following the procedure.

\section{Statistical analysis}

A power analysis was performed based on previous observations of changes in upper extremity strength $(24,25)$ using Fisher's exact test. Sample sizes of 52 in each group achieved $>80 \%$ power to detect a group difference in proportions, assuming that $20 \%$ of patients in the CIESI-L group would demonstrate a motor deficit meeting the MCID after the injection and those in the saline group would demonstrate no difference in motor strength. Additionally, this calculation included a $2 \%$ measurement error associated with the strength testing method described. ${ }^{20}$ The alpha level of this test statistic was set at 0.05. To account for possible attrition, we aimed to enroll 120 participants.

Descriptive statistics were calculated for patients' demographics as well as each outcome variable. Specifically, mean and SD were used for continuous variables, while frequency and proportion were used for categorical variables. Betweengroup comparisons of the demographic variables were made using (1) an independent t-test and (2) Pearson's $\chi^{2}$ test (or Fisher's exact test in case of low expected frequencies) for categorical variables. Preliminary analysis using the Shapiro-Wilk W-test ${ }^{23}$ showed that age and baseline NRS scores were approximately normally distributed $(\mathrm{p}>0.05)$, but BMI values were 
not $(p<0.001)$. Hence, BMI was analyzed using the Wilcoxon rank-sum test/Mann-Whitney two-sample statistic, ${ }^{24}{ }^{25}$ instead. Furthermore, proportions and 95\% CI were calculated for each binary outcome variable $(\geq 20 \%$ change in myotome, $\geq 50 \%$ NRS pain reduction, and $\geq 2$ point NRS change (MCID for neck pain)). ${ }^{26}$ Contingency table analysis and the Wilcoxon rank-sum test/Mann-Whitney two-sample statistic were performed for the binary outcome variables and the mean changes in NRS (as change scores on NRS in the lidocaine group were not normally distributed according to the Shapiro-Wilk W-test $(\mathrm{p}<0.001))$, respectively, by CIESI-L versus CIESI-S. In case there were significant differences in the demographic variables, multivariate logistic and linear regression analyzes were conducted to examine the binary and continuous outcome variables by group, while accounting for the statistically significant demographic variables. Finally, the pre-post changes in strengths for EF;C5 (left and right), WE;C6 (left and right), EX;C7 (left and right), and $\mathrm{HG} ; \mathrm{C} 8, \mathrm{~T} 1$ (dominant and non-dominant) myotomes by group (lidocaine versus saline) were examined, using the Wilcoxon rank-sum test/Mann-Whitney two-sample statistic, because of non-normal distributions of these variables based on the Shapiro-Wilk W-test $(\mathrm{p}<0.05)$.
Participants whose preinjection and postinjection NRS scores were zero were excluded from the analysis. Missing preprocedural NRS scores were excluded from the analysis. Missing preprocedural NRS or a pre-NRS and post-NRS score of zero prevents the ability to calculate an NRS difference. Those whose post-NRS scores were missing were considered non-responders such that they were conservatively assumed to not achieve meaningful pain relief.

\section{RESULTS}

One hundred fifty-nine patients were approached to participate in this clinical trial and 123 met inclusion criteria and were enrolled and randomized. Three participants were withdrawn from the study and 120 participants were included in the final data analysis (figure 1). Baseline demographic, clinical, symptom laterality, and characteristics were collected and summarized (table 1). There were no statistically significant group differences in all variables $(p>0.05)$, except for BMI $(p=0.007)$ and physical examination weakness in any myotome $(p=0.016)$.

\section{Primary outcome}

Postprocedural weakness of $\geq 20 \%$ (MCID) was observed in 25 of $60(41.7 \%)$ participants in the CIESI-L group and 30 of 60

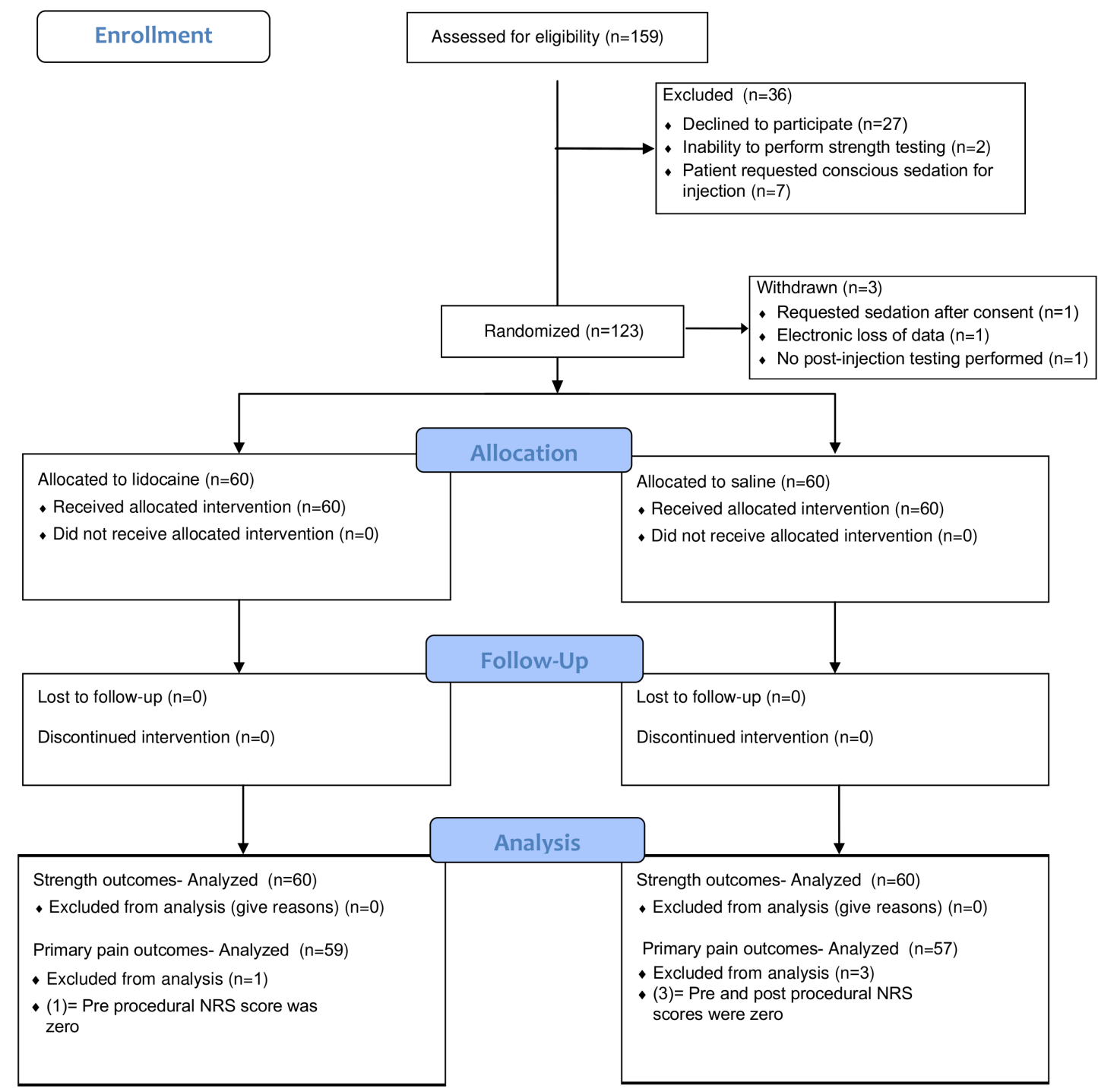

Figure 1 Consort 2010 flow diagram. NRS, numerical rating scale. 


\begin{tabular}{|c|c|c|c|}
\hline \multirow[b]{2}{*}{ Variable } & \multirow{2}{*}{$\begin{array}{l}\begin{array}{l}\text { Lidocaine diluent } \\
\text { group }\end{array} \\
(n=60)\end{array}$} & \multirow{2}{*}{$\begin{array}{l}\text { Saline diluent } \\
\text { group } \\
(n=60)\end{array}$} & \multirow[b]{2}{*}{$P$ value } \\
\hline & & & \\
\hline Age (years) & $56.6 \pm 14.0$ & $56.3 \pm 13.4$ & $0.926^{*}$ \\
\hline BMI $\left(\mathrm{kg} / \mathrm{m}^{2}\right)$ & $26.5 \pm 4.9$ & $29.2 \pm 6.1$ & $0.007 \dagger$ \\
\hline Baseline NRS & $4.9 \pm 2.3$ & $5.4 \pm 2.4$ & $0.257^{*}$ \\
\hline \multicolumn{4}{|c|}{ Gender, freq (\%) } \\
\hline Male & $30(50.0)$ & $26(43.3)$ & $0.464 \ddagger$ \\
\hline Female & $30(50.0)$ & $34(56.7)$ & \\
\hline \multicolumn{4}{|c|}{ Radicular pain, freq (\%) } \\
\hline C5 & $15(25.0)$ & $13(21.7)$ & $0.826 \S$ \\
\hline C6 & $29(48.3)$ & $30(50.0)$ & \\
\hline C7 & $12(20.0)$ & $9(15.0)$ & \\
\hline C8 & 0 & $1(1.7)$ & \\
\hline Missing & $4(6.7)$ & $7(11.6)$ & \\
\hline \multicolumn{4}{|c|}{ Laterality of symptoms, freq (\%) } \\
\hline Unilateral & $48(78.3)$ & $48(80.0)$ & $0.634 \ddagger$ \\
\hline Bilateral & $13(21.3)$ & $12(20.0)$ & \\
\hline Missing & $1(1.7)$ & $1(1.7)$ & \\
\hline \multicolumn{4}{|c|}{$\begin{array}{l}\text { Physical exam - Weakness in any myotomeף, freq } \\
(\%)\end{array}$} \\
\hline Yes & $3(5.0)$ & $12(20.0)$ & $0.016 \ddagger$ \\
\hline No & $54(90.0)$ & $47(78.3)$ & \\
\hline Missing & $3(5.0)$ & $1(1.7)$ & \\
\hline
\end{tabular}

Values are mean (SD), unless specified otherwise.

*From independent t-test.

tFrom the Wilcoxon rank-sum test/Mann-Whitney two-sample statistic.

\#From Pearson $\chi^{2}$ test.

$\S$ From Fisher's exact test.

IWithout dynamometry

BMI, body mass index; NRS, numerical rating scale.

(50\%) participants in the CIESI-S group (RR 0.83 (95\% CI, 0.56 to 1.23)) (table 2).

\section{Secondary outcomes}

Postprocedural pain reduction of $\geq 50 \%$ was observed in 19 of $59(32.2 \%)$ participants in the CIESI-L group and 12 of 57 participants (21.1\%) in the CIESI-S group (RR 1.53 (95\% CI, 0.82 to 2.86)) (table 2). Two participants in the CIESI-L and four in the CIESI-S groups were excluded from the analysis because either preprocedural NRS was missing or pre-NRS and post-NRS scores were zero.

\section{Adverse outcomes}

There were no severe adverse outcomes in any participants of either group. Vasovagal symptoms, increased pain at injection site, and paresthesia/numbness were noted in three (2.5\%), two $(1.7 \%)$, and six (5\%) participants during or immediately after the CIESI. There was no significant between-group difference (all RR 95\% CI crossed 1). Two (1.7\%) participants had asymptomatic, transient cardiac rhythm changes during CIESI; both participants were in the CIESI-L group. A summary of adverse events is noted in table 3 .

Phone calls were made approximately 1 day after CIESI to evaluate postprocedural numbness and weakness or other adverse events. In the CIESI-L and CIESI-S groups, three of the $42(7.1 \%$ (95\% CI, 0.0 to 15.0$))$ and four of the $44(9.0 \%$ (95\% CI, 1.0 to 18.0)) reported 'mild' numbness. In the CIESI-L and CIESI-S groups, one of the $42(2.0 \%(95 \% \mathrm{CI}, 0.0$ to 7.0$))$ and three of the 44 (7.0\% (95\% CI, 0.0 to 14.0)) reported 'mild' weakness. The subjective 'mild' weakness was not verified by dynamometry.

\section{Subanalysis}

Regarding strength, $68.3 \%$ (95\% CI, 59.4 to 76.1 ) of all patients showed $\geq 20 \%$ strength improvement in $\geq 1$ myotome, and $24.2 \%$ (95\% CI, 17.3 to 32.7 ) showed both a $\geq 20 \%$ strength improvement and a $\geq 20 \%$ strength decrease in $\geq 2$ different myotomes. We identified a pain NRS reduction of $\geq 2$ points (MCID) from preinjection to postinjection in $35.6 \%$ of all participants with no significant between-group difference (RR 1.33 (95\% CI, 0.81 to 2.18$)$ ). Similarly, the mean pain reductions were not significantly different between the CIESI-L and CIESI-S groups $(1.5 \pm 2.0$ vs $0.9 \pm 2.0, p=0.168)$. We found no significant effect of lidocaine on any of the binary outcome variable compared with saline (table 4).

Since there were significant group differences in BMI and physical examination weakness in any myotome as reported above, multiple logistic and linear regression models were used to examine the binary ( $\geq 20 \%$ change in myotome, $\geq 50 \% \mathrm{NRS}$ pain reduction, and $\geq 2$ point NRS change (MCID for neck pain)) and continuous (mean changes in NRS) outcome variables by group (lidocaine versus saline), while adjusting for BMI and physical examination weakness. The analysis showed that there

Table 2 Primary and secondary outcomes

\begin{tabular}{|c|c|c|c|c|}
\hline \multirow{2}{*}{$\begin{array}{l}\text { Primary outcome } \\
\text { Group }\end{array}$} & \multicolumn{2}{|c|}{$\geq 20 \%$ Weakness in $\geq 1$ myotome } & \multirow[b]{2}{*}{ Risk $(95 \% \mathrm{Cl})^{*}$} & \multirow[b]{2}{*}{$\operatorname{RR}(95 \% \mathrm{Cl}) \dagger$} \\
\hline & Yes & No & & \\
\hline Lidocaine & $25(41.7)$ & $35(58.3)$ & 41.7 (29.8 to 54.5$)$ & 0.83 (0.56 to 1.23$)$ \\
\hline Saline & $30(50.0)$ & $30(50.0)$ & $50.0(37.5$ to 62.5$)$ & \\
\hline Total & $55(45.8)$ & $65(54.2)$ & 45.8 (37.1 to 54.9$)$ & \\
\hline Secondary outcome & $\geq 50 \%$ Pai & & & \\
\hline Lidocaine & $19(32.2)$ & $40(67.8)$ & $32.2(20.3$ to 44.1$)$ & 1.53 (0.82 to 2.86$)$ \\
\hline Saline & $12(21.1)$ & $45(78.9)$ & 26.7 (13.8 to 39.6$)$ & \\
\hline Total & $31(26.7)$ & $85(73.3)$ & 26.7 (18.7 to 34.8$)$ & \\
\hline
\end{tabular}

Values are frequency (\%) unless specified otherwise. P value from Pearson's $\chi^{2}$ test.

Patients whose baseline NRS scores were missing or zero were excluded from the analysis.

Patients whose post-NRS scores were missing were considered treatment failure (ie, $\%$ change $=0 \%$ and $50 \%$ pain reduction=no).

Patients whose baseline and post-NRS scores were both zero were excluded from the analysis.

${ }^{*}$ Risk $(\%)=($ freq (yes) / freq (total) $) \times 100$

tRelative risk=risk (lidocaine) / risk (saline)

freq, frequency; NRS, numerical rating scale; RR, relative risk. 
Original research

\begin{tabular}{|c|c|c|c|c|c|}
\hline \multirow{2}{*}{$\begin{array}{l}\text { Outcome } \\
\text { Group }\end{array}$} & \multicolumn{2}{|c|}{ Vasovagal } & \multirow[b]{2}{*}{ Risk $(95 \% \mathrm{Cl})^{*}$} & \multirow[b]{2}{*}{$\operatorname{RR}(95 \% \mathrm{Cl}) \dagger$} & \multirow[b]{2}{*}{$P$ value $\neq$} \\
\hline & Yes & No & & & \\
\hline Lidocaine & $1(1.7)$ & $59(98.3)$ & $1.7(0.0$ to 5.0$)$ & 0.50 (0.05 to 5.37$)$ & 0.999 \\
\hline Saline & $2(3.3)$ & $58(96.7)$ & $3.3(0.0$ to 8.0$)$ & & \\
\hline Total & $3(2.5)$ & $117(97.5)$ & $2.5(0.0$ to 5.0$)$ & & \\
\hline Outcome & \multicolumn{2}{|c|}{ Cardiac rhythm changes } & & & \\
\hline Lidocaine & $2(3.3)$ & $58(96.7)$ & $3.3(0.0$ to 8.0$)$ & Unable to calculate & 0.496 \\
\hline Saline & $0(0.0)$ & $60(100.0)$ & $0.0(0.0$ to 0.0$)$ & & \\
\hline Total & $2(1.7)$ & $118(98.3)$ & $1.7(0.0$ to 4.0$)$ & & \\
\hline Outcome & \multicolumn{2}{|c|}{ Pain at injection site } & & & \\
\hline Lidocaine & $1(1.7)$ & $59(98.3)$ & $1.7(0.0$ to 5.0$)$ & 1.00 (0.06 to 15.62$)$ & 0.999 \\
\hline Saline & $1(1.7)$ & $59(98.3)$ & $1.7(0.0$ to 5.0$)$ & & \\
\hline Total & $2(1.7)$ & $118(98.3)$ & $1.7(0.0$ to 4.0$)$ & & \\
\hline Outcome & \multicolumn{2}{|c|}{ Paresthesia/numbness } & & & \\
\hline Lidocaine & $2(3.3)$ & $58(96.7)$ & $3.3(0.0$ to 8.0$)$ & 0.50 (0.10 to 2.63$)$ & 0.679 \\
\hline Saline & $4(6.7)$ & $56(93.3)$ & $6.7(0.0$ to 13$)$ & & \\
\hline Total & $6(5.0)$ & $114(95.0)$ & $5.0(1.0$ to 9.0$)$ & & \\
\hline
\end{tabular}

Values are frequency (\%) unless specified otherwise.

${ }^{*}$ Risk $(\%)=($ freq (yes) / freq (total) $) \times 100$

tRelative risk=risk (lidocaine) / risk (saline)

$\ddagger P$ value from Fisher's exact test.

freq, frequency; RR, relative risk.

was no group effect on any of the outcome variables mentioned previously ( $\mathrm{p}=0.688,0.329,0.240$, and 0.137 , respectively). Hence, the significant group differences in BMI and physical examination weakness in any myotome did not change the overall results. The pre-post changes in strengths for EF; C5 (left and right), WE;C6 (left and right), EX;C7 (left and right), and HG;C8,T1 grip (dominant and non-dominant) myotomes by group (lidocaine versus saline) are summarized in table 5 . There were no significant group differences in the pre-post changes in these measures between the two groups $(\mathrm{p}>0.05)$.

\section{DISCUSSION}

We report a novel double-blinded, randomized trial comparing the incidence of clinically meaningful changes in strength and pain after CIESI with lidocaine compared with saline used as the epidural injectate diluent. We previously published a single-arm cohort study demonstrating that $20 \%$ of participants experience a $\geq 20 \%$ decrease in strength in at least one myotome as measured by dynamometry after CIESI with $1.0 \mathrm{~mL}$ of $1 \%$ lidocaine used as the diluent. ${ }^{15}$ In the present study, a higher dose of lidocaine was intentionally used: $2.0 \mathrm{~mL}$ of $1 \%$ lidocaine for a total injectate volume of $4 \mathrm{~mL}$. Despite this higher dose of lidocaine, and surprisingly counter to our hypothesis, $41.7 \%$ and $50.0 \%$ of participants in the CIESI-L and CIESI-S groups, respectively, experienced $a \geq 20 \%$ decrease in strength in at least one myotome between 20 and $30 \mathrm{~min}$ postinjection, resulting in no between-group difference (RR 0.83 (95\% CI, 0.56 to 1.23$)$ ). Also unexpectedly, we found no significant difference in the proportion of participants who experienced $>50 \%$ pain reduction in the CIESI-L and CIESI-S groups (RR 1.53 (95\% CI, 0.82 to 2.86)). These findings indicate that patients undergoing CIESI

\begin{tabular}{|c|c|c|c|c|c|}
\hline \multirow{2}{*}{$\begin{array}{l}\text { Outcome } \\
\text { Group }\end{array}$} & \multicolumn{2}{|c|}{$\geq 20 \%$ Strength improvement in $\geq 1$ myotome } & \multirow[b]{2}{*}{ Risk $(95 \% \mathrm{Cl})^{*}$} & \multirow[b]{2}{*}{ RR $(95 \% \mathrm{Cl}) \dagger$} & \multirow[b]{2}{*}{$P$ value $\neq$} \\
\hline & Yes & No & & & \\
\hline Lidocaine & $44(73.3)$ & $16(26.7)$ & $73.3(60.7$ to 83.1$)$ & $1.16(0.91$ to 1.48$)$ & 0.239 \\
\hline Saline & $38(63.3)$ & $22(36.7)$ & $63.3(50.4$ to 74.6$)$ & & \\
\hline Total & $82(68.3)$ & $38(31.7)$ & $68.3(59.4$ to 76.1$)$ & & \\
\hline Outcome & \multicolumn{5}{|c|}{$\geq 20 \%$ Weakness and strength change in $\geq 2$ myotomes } \\
\hline Lidocaine & $14(23.3)$ & $46(76.7)$ & $23.3(14.3$ to 35.8$)$ & 0.93 (0.49 to 1.76$)$ & 0.831 \\
\hline Saline & $15(25.0)$ & $45(75.0)$ & $25.0(15.6$ to 37.6$)$ & & \\
\hline Total & $29(24.2)$ & $91(75.8)$ & $24.2(17.3$ to 32.7$)$ & & \\
\hline Outcome & \multicolumn{5}{|c|}{$\geq 2$ on NRS (MCID) } \\
\hline Lidocaine & $24(40.1)$ & $35(59.3)$ & $40.7(28.1$ to 53.2$)$ & $1.33(0.81$ to 2.18$)$ & 0.249 \\
\hline Saline & $18(30.5)$ & $41(69.5)$ & 30.51 (18.8 to 42.3 ) & & \\
\hline Total & 42 (35.6) & 76 (64.4) & 35.6 (27.0 to 44.2 ) & & \\
\hline
\end{tabular}

Values are frequency (\%) unless specified otherwise.

${ }^{*}$ Risk $(\%)=($ freq (yes) / freq (total) $) \times 100$

tRelative risk=risk (lidocaine) / risk (saline)

$\ddagger P$ value from Pearson's $\chi^{2}$ test.

freq, frequency; MCID, minimal clinically important difference; NRS, numerical rating scale; RR, relative risk. 
Table 5 Between-group pre-post procedural strength difference by myotome

\begin{tabular}{|c|c|c|c|c|c|c|c|}
\hline \multirow[b]{2}{*}{ Myotome } & \multicolumn{3}{|l|}{ Lidocaine } & \multicolumn{3}{|l|}{ Saline } & \multirow[b]{2}{*}{ P value* } \\
\hline & Pre & Post & Difference & Pre & Post & Difference & \\
\hline Grip (dominant) & $28.2(10.0)$ & $27.8(10.4)$ & $-0.4(3.8)$ & $26.5(11.9)$ & $25.9(12.1)$ & $-0.6(4.4)$ & 0.869 \\
\hline Grip (non-dominant) & $27.6(10.4)$ & $26.8(10.6)$ & $-0.8(4.3)$ & $24.9(11.5)$ & $24.3(11.4)$ & $-0.6(4.0)$ & 0.939 \\
\hline Wrist extension (left) & $3.6(1.9)$ & $4.1(2.4)$ & $0.5(0.9)$ & $3.2(1.9)$ & $3.9(3.2)$ & $0.7(2.1)$ & 0.611 \\
\hline Wrist extension (right) & $3.4(1.8)$ & $3.9(2.3)$ & $0.5(1.1)$ & $3.3(2.3)$ & $3.8(3.3)$ & $0.5(1.6)$ & 0.44 \\
\hline Elbow extension (left) & $10.3(3.9)$ & $10.5(4.7)$ & $0.2(1.7)$ & $9.3(4.4)$ & $9.8(4.6)$ & $0.5(2.4)$ & 0.401 \\
\hline Elbow extension (right) & $10.1(4.3)$ & $10.6(4.9)$ & $0.5(1.8)$ & $9.4(5.9)$ & $10.1(7.3)$ & $0.7(2.6)$ & 0.577 \\
\hline Elbow flexion (left) & $13.2(6.8)$ & $13.1(6.6)$ & $-0.1(2.3)$ & $12.8(9.0)$ & $13.2(10.5)$ & $0.4(4.2)$ & 0.512 \\
\hline Elbow flexion (right) & $12.8(6.9)$ & $12.8(6.2)$ & $0.0(2.6)$ & $13.4(11.5)$ & $13.3(11.2)$ & $-0.1(3.9)$ & 0.374 \\
\hline
\end{tabular}

* $P$ values from the Wilcoxon rank-sum test/Mann-Whitney two-sample statistic.

may experience clinically significant weakness after the injection and no immediate improvement in pain regardless of use of a lidocaine or a saline diluent.

These results have important safety implications. Upper extremity weakness appears to result in $40 \%$ to $50 \%$ of patients who undergo CIESI regardless of diluent. It is unclear from our study how long this weakness lasts. Transient upper extremity weakness, regardless of diluent, should be discussed with all patients during the informed consent process and at discharge. Particular attention should be given to those who are dependent on upper extremity strength for safe ambulation (holding a rail, gait aid use, and so on) to avoid falls following a CIESI. Furthermore, although not observed as an adverse event in our study population, using local anesthetic as a diluent may put patients at increased risk of HSB, ${ }^{9-11} 13$ without evidence of greater postinjection immediate pain reduction. While an analgesic benefit is associated with higher doses of epidural local anesthetic, these increased doses also increase the risk of more profound motor block.

It is unclear why more substantial group differences in strength post-CIESI in the lidocaine group were not observed. Indeed, the concentration and volume of lidocaine used here may not be significant enough to produce a meaningful effect. Although user error with dynamometer muscle testing is possible, similar study protocols have been validated in other studies. ${ }^{16-21}$ Conversely, the validation studies were performed in non-antalgic participants. It is unknown how baseline or postprocedural pain affects the validity of dynamometer testing. Pain-related weakness or 'pain inhibition' may also be a relevant factor in strength testing. In the present study, approximately $60 \%$ of all participants experienced a strength reduction or improvement in at least one myotome by the established MCID.

The lack of immediate therapeutic benefit of lidocaine is likely the result of a dilutional effect. One study revealed that $2 \mathrm{~mL}$ of injectate will spread as far cephalad as C3 with an interlaminar injection at C6-7 or C7-T1. ${ }^{27}$ While an inappropriate time of re-assessment postinjection (too soon or too long) could obscure observation of the anesthetic effect on strength, this is unlikely to be the case in the present study given the timed adherence of the study protocol for strength testing. Other investigations have demonstrated that maximal nerve block occurs at $15 \pm 3 \mathrm{~min}$ after injection with $1 \%$ lidocaine, ${ }^{28}$ with effects lasting up to 3 hours. $^{29}$ In the current study, postprocedural NRS scores were measures at approximately $20 \mathrm{~min}$. The between-group difference may have been reduced if pain reduction occurred by mechanical flushing of chemical inflammatory mediators and not by anesthetizing sensory fibers. ${ }^{30}$

\section{Strength and limitations}

There are many strengths of this study, including study design, blinding, low risk of bias, generalizability, statistical power, and objective and clinically relevant outcomes. One of the weaknesses of this study is the duration of outcome assessment. Postprocedural strength was not assessed beyond $30 \mathrm{~min}$. Another limitation of this study is the pain reduction outcome. Pain and pain-related outcomes are subjective and variable. One inclusion criteria for the current study was a screening NRS value $\geq 4$; however, screening values were not recorded. The secondary pain outcomes were calculated based on preprocedural (immediately before) and postprocedural NRS scores. As a result, four participants had pre-CIESI NRS scores of zero, making NRS percentage change mathematically impossible. As with all pain outcome studies, the 'typical' pain should be targeted and measured. It may have been beneficial to include a validated patient-reported outcome such as the patient's global impression of change (PGIC). If there was a significant difference between NRS and PGIC, the use of low-dose lidocaine in CIESI may be justified.

\section{Future studies}

High-quality studies evaluating the validity of dynamometry used to test strength in patients with pain conditions, duration of postprocedural strength changes, and the patient-perceived therapeutic effect of low-dose lidocaine warrant further research.

\section{CONCLUSION}

The present data indicate that low-dose lidocaine compared with saline used as a diluent in CIESI does not significantly increase the risk of transient post-CIESI myotomal upper extremity weakness but also does not substantially increase the likelihood of immediate, clinically meaningful pain relief. As such, the use of low-dose lidocaine may provide minimal advantage to saline, yet carries the risk of HSB on unintended intrathecal administration.

Contributors ZD, RWK, and DW conceived and designed the study; ZD, SC, DB, and DW were involved in data acquisition; ZD, TB, RWK, MT, and DW were involved in analysis and/or interpretation of data; ZD, TB, and DW drafted the manuscript; ZD, TB, SC, RWK, DB, MT, and DW revised the manuscript critically for important intellectual content. All authors gave approval for the final manuscript.

Funding The authors have not declared a specific grant for this research from any funding agency in the public, commercial or not-for-profit sectors.

Competing interests ZM and MD serve on the Board of Directors of the Spine Intervention Society.

Patient consent for publication Not required.

Ethics approval The study protocol was approved by the Northwestern University Institutional Review Board (IRB STU00204980). 
Provenance and peer review Not commissioned; externally peer reviewed.

Data availability statement All data relevant to the study are included in the article or uploaded as supplementary information. Data is available upon request.

\section{REFERENCES}

1 Radhakrishnan K, Litchy WJ, O'Fallon WM, et al. Epidemiology of cervical radiculopathy. Brain 1994;117:325-35.

2 Peloso PMJ, Gross A, Haines T, et al. Withdrawn: medicinal and injection therapies for mechanical neck disorders. Cochrane Database Syst Rev 2015:CD000319.

3 House LM, Barrette K, Mattie R, et al. Cervical epidural steroid injection. Phys Med Rehabil Clin N Am 2018;29:1-17.

4 Schneider B, Zheng P, Mattie R, et al. Safety of epidural steroid injections. Expert Opin Drug Saf 2016;15:1031-9.

5 Bogduk N. Practice guidelines for spinal diagnostic and Trement procedures. 2nd ed, 2013

6 Vydra D, McCormick Z, Clements N, et al. Current trends in steroid dose choice and frequency of administration of epidural steroid injections: a survey study. $P m R$ 2020;12:49-54.

7 Clements N, Vydra D, Cushman DM, et al. Trends in steroid agent and diluent choices for epidural steroid injections: a survey of spine intervention Society physicians. Reg Anesth Pain Med 2019;44:809-13.

8 Bellini M, Barbieri M. Systemic effects of epidural steroid injections. Anestezjol Intens Ter 2013;45:93-8.

9 Chung SG. Convulsion caused by a lidocaine test in cervical transforaminal epidural steroid injection. Pm R 2011;3:674-7.

10 Mahli A, Coskun D, Akcali DT. Aetiology of convulsions due to stellate ganglion block: a review and report of two cases. Eur J Anaesthesiol 2002;19:376-80.

11 Capdevila X, Biboulet P, Rubenovitch J, et al. The effects of cervical epidural anesthesia with bupivacaine on pulmonary function in conscious patients. Anesth Analg 1998;86:1033-8.

12 Bansal S, Turtle MJ. Inadvertent subdural spread complicating cervical epidural steroid injection with local anaesthetic agent. Anaesth Intensive Care 2003;31:570-2.

13 Collier CB. Accidental subdural block: four more cases and a radiographic review. Anaesth Intensive Care 1992;20:215-25.

14 Karasek M, Bogduk N. Temporary neurologic deficit after cervical transforaminal injection of local anesthetic. Pain Med 2004;5:202-5.
15 McCormick ZL, Nelson A, Kendall MC, et al. Does cervical interlaminar epidural steroid injection with low-dose lidocaine cause objective upper extremity weakness? A preliminary study. Pain Med 2017;18:2296-305.

16 Bohannon RW. Reference values for extremity muscle strength obtained by hand-held dynamometry from adults aged 20 to 79 years. Arch Phys Med Rehabil 1997;78:26-32.

17 Bohannon RW. Grip strength impairments among older adults receiving physical therapy in a home-care setting. Percept Mot Skills 2010:111:761-4.

18 Hayes K, Walton JR, Szomor ZL, et al. Reliability of 3 methods for assessing shoulder strength. J Shoulder Elbow Surg 2002;11:33-9.

19 Kolber MJ, Beekhuizen K, Cheng M-SS, et al. The reliability of hand-held dynamometry in measuring isometric strength of the shoulder internal and external rotator musculature using a stabilization device. Physiother Theory Pract 2007;23:119-24.

20 Awatani T, Mori S, Shinohara J, et al. Same-session and between-day intra-rater reliability of hand-held dynamometer measurements of isometric shoulder extensor strength. J Phys Ther Sci 2016;28:936-9.

21 Fieseler G, Molitor T, Irlenbusch L, et al. Intrarater reliability of goniometry and handheld dynamometry for shoulder and elbow examinations in female team handball athletes and asymptomatic volunteers. Arch Orthop Trauma Surg 2015;135:1719-26.

22 Dworkin RH, Turk DC, Farrar JT, et al. Core outcome measures for chronic pain clinical trials: IMMPACT recommendations. Pain 2005;113:9-19.

23 Royston JP. A Simple Method for Evaluating the Shapiro-Francia W' Test of NonNormality. Stat, 1983.

24 Mann HB, Whitney DR. On a test of whether one of two random variables is stochastically larger than the other. Ann Math Stat 1947;18:50-60.

25 Wilcoxon F. Individual comparisons by ranking methods. Biometrics Bulletin $1945 ; 1: 80$

26 Kovacs FM, Abraira V, Royuela A, et al. Minimum detectable and minimal clinically important changes for pain in patients with nonspecific neck pain. BMC Musculoskelet Disord 2008:9:43.

27 Goel A, Pollan JJ. Contrast flow characteristics in the cervical epidural space. Spine 2006;31:1576-9.

28 Atanassoff PG, Kelly DJ, Ayoub CM, et al. Electromyographic assessment of ulnar nerve motor block induced by lidocaine. J Clin Anesth 1998;10:641

29 Rule AM. American Society of health-system pharmacists' pain management network. J Pain Palliat Care Pharmacother 2004;18:59-62.

30 Mulleman D, Mammou S, Griffoul I, et al. Pathophysiology of disk-related sciatica. I.-Evidence supporting a chemical component. Jt Bone Spine 2006;73:151-8. 